\title{
Assessment of the Impact of Tardive Dyskinesia in Clinical Practice: Consensus Panel Recommendations
}

\author{
Richard Jackson' \\ Matthew N Brams ${ }^{2}$ \\ Leslie Citrome $\mathbb{I D}^{3}$ \\ Amber R Hoberg ${ }^{4}$ \\ Stuart $\mathrm{H}$ Isaacson ${ }^{5}$ \\ John M Kane (iD) ${ }^{6}$ \\ Rajeev Kumar $^{7}$ \\ 'University of Michigan School of \\ Medicine, Ann Arbor, MI, USA; ${ }^{2}$ Baylor \\ College of Medicine, Houston, TX, USA; \\ ${ }^{3}$ New York Medical College, Valhalla, NY, \\ USA; ${ }^{4}$ GeroPsych Associates of Central \\ Texas, Austin, TX, USA; ${ }^{5}$ Parkinson's \\ Disease and Movement Disorders Center \\ of Boca Raton, Boca Raton, FL, USA; \\ ${ }^{6}$ Feinstein Institution for Medical \\ Research, Lynbrook, NY, USA; ${ }^{7}$ Rocky \\ Mountain Movement Disorders Center \\ Englewood, CO, USA
}

Purpose: Tardive dyskinesia (TD) is a hyperkinetic movement disorder in which patients experience abnormal involuntary movements that can have profound negative impacts on physical, cognitive, and psychosocial functioning. Use of measures to assess the functional impact of TD in routine clinical practice is lacking. To address this gap, an advisory panel of experts in psychiatry and movement disorder neurology was convened to develop consensus recommendations on assessment of the impact of TD on patients' functioning that can be used in clinical practice.

Methods: An advisory panel provided recommendations using an iterative process, beginning with a narrative literature review regarding current practices for assessing the impact of TD in clinical settings. A detailed summary was generated, and the advisory panel provided comments about the content and answered questions about assessing TD impact in clinical practice. The panelists' responses were discussed during a virtual meeting held on August 28, 2020. A second meeting on September 25, 2020, focused on developing and refining recommendations for assessment of the impact of TD in clinical practice. At the conclusion of the second meeting, general consensus was reached on all recommendation statements.

Results: As part of routine clinical practice, it is imperative to assess the impact of TD on the patient's life to help guide treatment decisions. Key domains for assessing the overall impact of TD include social, physical, vocational, and psychological functioning and the impact of TD on the underlying psychiatric disorder. Assessment of TD impact should be performed at every patient visit. Impact assessments should include consultation with patients, caregivers, and family members. Shared decision-making to initiate TD treatment should consider impact.

Conclusion: The impact of TD should be assessed routinely, including the key domains of social, physical, vocational, and psychological functioning and the impact of TD on the underlying psychiatric disorder.

Keywords: hyperkinetic movement, functional domains, diagnosis, treatment

\section{Introduction}

Tardive dyskinesia (TD) is a hyperkinetic movement disorder in which patients experience abnormal involuntary movements that occur most often in the orofacial region but can also occur in the neck, trunk, upper and lower extremities, and other muscles (eg, diaphragmatic and pharyngeal musculature). ${ }^{1-5}$ TD can occur during or after stopping treatment with dopamine receptor antagonists or partial agonists, including typical (first-generation) and atypical (second-generation) antipsychotics, and some medications used to treat gastrointestinal disorders, such as metoclopramide. $^{3,6-8}$ The lifetime prevalence of TD in patients treated with
Correspondence: Richard Jackson University of Michigan School of Medicine, 4III Andover Road, Suite WI00, Bloomfield Hills, MI, 48302, USA

Tel +I 2482905400

$\mathrm{Fax}+|248290540|$

Email richardjackson2000@yahoo.com 
antipsychotics is estimated to be between approximately $15 \%$ and $40 \%$, with higher rates reported in older (age $>55$ years) patients ${ }^{9}$; higher rates are also observed among women who are postmenopausal. ${ }^{6,10}$

TD can have a profound negative impact on patients' day-to-day functioning and may lead to negative physical, cognitive, and psychosocial outcomes. ${ }^{11,12} \mathrm{TD}$ underdiagnosis, lack of awareness that all firstand second-generation antipsychotics have the potential to cause TD, and lack of generally accepted and adopted objective measures for the accurate clinical assessment of the impact of TD are current shortcomings in managing care for individuals with TD. ${ }^{10}$ Although several different instruments have been suggested or preliminarily tested for assessing TD impact (eg, the modified Craniocervical Dystonia Questionnaire [mCDQ-24], various performance- and cognition-based assessments, the Tardive Dyskinesia Rating Scale [TDRS], the Dyskinesia Identification System: Condensed User Scale [DISCUS], and items 9 and 10 of the Abnormal Involuntary Movement Scale [AIMS]), clinicians have expressed resistance or lack of time for utilizing such scales to assess TD impact. ${ }^{12-18}$ Furthermore, no single tool is adequate to evaluate the potentially broad overall impact of TD on functioning.

Effectively assessing the impact of TD is essential for clinicians as they determine whether and how urgently to treat TD and to develop an individualized plan for treatment optimization that will reduce the impact of TD on the patient's life. To address the gap in current guidance on the assessment of TD impact, an advisory panel was convened with the goal of establishing consensus recommendations on the importance of key elements in assessing the impact of TD on patients' functioning that can be used in routine clinical practice.

\section{Materials and Methods}

The advisory panel for this project consisted of 7 individuals with clinical and/or research expertise in TD, with specialties in psychiatry (R. Jackson [chair], M.N. Brams, L. Citrome, A.R. Hoberg, and J.M. Kane) or movement disorder neurology (S.H. Isaacson and R. Kumar). Ethical approval and informed consent were not applicable.

A narrative literature review was conducted to summarize what has been published on approaches to assessing the impact of TD in clinical research and clinical practice, which would serve as a foundation for the consensus panel to generate best practice recommendations for assessing the impact of TD in clinical practice. The literature searches and preparation of the narrative summary were performed by Interactive Forums, Inc. (Conshohocken, PA) under the guidance and direction of the panel chair, Richard Jackson, MD.

The initial literature search was performed in June 2020 utilizing the US National Library of Medicine PubMed.gov database to identify relevant Englishlanguage review articles published in the 5 years prior to May 31, 2020. Search terms included "tardive dyskinesia" AND "diagnosis," "assessment," "prevalence," "risk factors," "outcomes," "clinical management," "impact assessment," "social impact," "functional impact," "quality of life," "psychological functioning," OR "cognition." Additional relevant publications (including several predating the most recent 5 years) were identified in the reference lists of pertinent review articles and by ad hoc PubMed and Google Scholar searches; other data sources included relevant posters from recent conference proceedings. The results of this literature review were provided to the advisors on July 17, 2020, and the advisors completed an assignment prior to Consensus Panel Meeting 1 that included providing detailed comments about the literature review summary and answering questions about assessing TD impact in clinical practice (see Supplementary Material). The panelists' responses were compiled for discussion during Consensus Panel Meeting 1.

The first of two virtual consensus panel meetings was held on August 28, 2020. This meeting was moderated by Stephen D. Lande, PhD (Interactive Forums, Inc.) and included discussion of the literature review summary, revision to this summary, and generation of recommendations about assessing TD impact in clinical practice. These recommendations were condensed into a series of statements that were distributed to the panel for review and feedback, which were collected before the second consensus panel meeting. As part of an assignment to be completed prior to Consensus Panel Meeting 2, advisors were asked to rate their level of agreement with each statement on a scale from 0 (not at all) to 4 (very much). This feedback showed that the mean level of agreement among the advisors $(\mathrm{n}=6)$ for each recommendation statement was high, with scores ranging from 3.0 to 4.0.

The second virtual consensus panel meeting was held on September 25, 2020, and was moderated by Dr Lande. This meeting included discussion and revision of the recommendations until a general consensus was reached on all statements. In total, recommendations were 
developed for six key topics: 1) diagnosis of TD, 2) importance of assessing the impact of TD, 3) key domains for assessing the impact of TD, 4) time points for assessing the impact of TD, 5) approaches to assessing the impact of TD, and 6) initiating treatment of TD (Box 1). The advisors determined objectives for the project moving forward, and all 7 participants expressed interest in participating in the development of this report, which presents the final consensus recommendations in context with evidence that guided the development of each statement.

\section{Results \\ Diagnosis of TD}

Recommendation \#1: Diagnosis of TD is determined based on dyskinetic movements in a patient with prior or current exposure to a dopamine receptor blocking agent. In clinical settings, observation of any persistent abnormal movements in one body area is sufficient for a diagnosis of TD after excluding other possible etiologies (eg, Huntington's disease or other drug-induced movement disorders). The individual may be unaware of the movements but still meet the criteria for a TD diagnosis.

An accurate TD diagnosis is crucial for all aspects of disease management for both patients and caregivers. ${ }^{19,20}$ An accurate diagnosis is also the foundation upon which all recommendations about assessing the impact of TD are based.

At the time of diagnosis, many patients present with what is considered to be "mild" TD based on assessment of abnormal movements. However, even mild dyskinetic movements can cause social anxiety and problems with daily activities, including eating, speaking, breathing, and ambulation. ${ }^{21,22}$ Because the term mild is subjective and patient experiences are variable, the advisory panel

Box I Recommendations for the Clinical Assessment of the Impact of Tardive Dyskinesia (TD)

\section{Diagnosis of TD}

- Diagnosis of TD is determined based on the presence of dyskinetic movements in a patient with prior or current exposure to a dopamine receptor blocking agent.

- In clinical settings, observation of any persistent abnormal movements in one body area is sufficient for a diagnosis of TD after excluding other possible etiologies (eg, Huntington's disease or other drug-induced movement disorders).

- The individual may be unaware of the movements but still meet the criteria for a TD diagnosis.

2. Importance of Assessing the Impact of TD

- It is imperative to assess the impact of TD on the patient's life in order to guide TD treatment recommendations and to aid in TD treatment optimization.

- The degree of TD impact influences the level of urgency with which symptoms should be addressed.

- The impact of the movements is multifactorial (eg, social withdrawal, stigmatization) and should not be determined based solely on severity of movement.

- Information should be garnered when possible from multiple sources, including family/caregivers and professional and nonprofessional staff.

3. Key Domains for Assessing the Impact of TD

- The following domains represent areas for assessing the overall impact of TD on an individual's life and function.

○ Social

- Physical

- Vocational

- Psychological

- Psychiatric

- The level of importance of each domain varies from patient to patient, depending upon their normal day-to-day activities, the location and severity of movements, and the impact reported by the individual combined with the impact perceived by others.

4. Time Points for Assessing the Impact of TD

- Assessment of TD impact should be a routine aspect of the evaluation performed at every patient visit because TD impact varies over time and can influence treatment decisions.

5. Approaches to Assessing the Impact of TD

- The key domains listed in Recommendation \#3 should be utilized to assess TD impact.

- When assessing TD impact, clinicians should consult with the patient directly and/or with caregivers/family/friends, especially when the individual has limited insight into movements and impact.

6. Initiating Treatment of TD

- A shared decision to initiate treatment of TD, as well as the urgency with which to initiate treatment, should consider the degree of impact. 
recommends against including the word mild when making a TD diagnosis. The panel also recommends against using the term "probable TD," which is a diagnostic category within the Schooler-Kane criteria for the diagnosis of TD in research settings ${ }^{19,23}$ but is not suitable for clinical settings as the use of this term may lead to underdiagnosis and undertreatment.

\section{Importance of Assessing the Impact of TD}

Recommendation \#2: It is imperative to assess the impact of TD on the patient's life to guide TD treatment recommendations and to aid in TD treatment optimization. The degree of TD impact may influence the level of urgency with which symptoms should be addressed. The impact of the movements is multifactorial (eg, social withdrawal and stigmatization) and should not be determined based solely on severity of movement. Information should be garnered when possible from multiple sources, including family/ caregivers and professional and nonprofessional staff.

When assessing the impact of TD on a patient's functioning, all individuals, including family members, caregivers, health professionals, and nonprofessionals, can provide valuable insight into how the patient is affected. A multidisciplinary approach should be adopted for comprehensive TD management. $^{24,25}$ Professionals who should be consulted may include psychiatrists, neurologists, general practitioners, pharmacists, nursing assistants, front office staff, case managers, social workers, home health aides, and physical, occupational, and recreational therapists. Ideally, all members of a multidisciplinary care team can contribute to assessment of the impact of TD. ${ }^{25}$ Because TD symptoms can fluctuate during each day and over time, caregivers and family members are often in the best position to observe changes in movements and impact that may not be observed during a clinical examination of the patient. $^{22}$

Patients may not spontaneously provide details related to the impact of TD; thus, it is crucial for clinicians to directly ask about disease impact. ${ }^{26,27}$ Some patients may be reluctant to discuss TD symptoms and impact with their care team because they fear that their physician may recommend discontinuation of a medication needed to manage underlying psychiatric disease. ${ }^{28}$ Others may be unable to accurately recognize and describe TD-related symptoms and impact. ${ }^{26}$ Lack of awareness of TD decreases the likelihood that patients will voluntarily mention abnormal movements at clinical visits. ${ }^{4}$ Thus, patient education about risk and signs of TD should be an integral part of the initiation of any antipsychotic medication and is part of the routine informed consent process when prescribing.

TD has been associated with decreased quality of life, increased hospitalizations, speech and eating difficulties, cognitive dysfunction, decreased employment, social withdrawal, stigmatization, amplified emotional distress, and increased psychopathology in the underlying psychiatric disorder when patients with TD are compared with nonTD counterparts. ${ }^{2,11,12,29-31}$ Although causality has not been established, and TD may be a marker of more severe illness for some patients, these potential negative effects underscore the importance of assessing TD and its impact.

\section{Key Domains for Assessing the Impact of TD}

Recommendation \#3: The following domains represent areas for assessing the overall impact of TD on an individual's life and function. The level of importance of each domain varies from patient to patient, depending upon their normal day-to-day activities, the location and severity of movements, and the impact reported by the individual combined with the impact perceived by others. They may also change in relevant importance over time.

(A) Social (eg, effect on how the patient is perceived by others, effect on relationships and interactions, avoidance of family and friends, avoidance of appearing in public, isolation, withdrawal, rejection by others)

(B) Physical (eg, biting tongue or inside of mouth, difficulty breathing, slurring of speech, difficulty eating/chewing/swallowing, reduced fine motor function/difficulty dressing with buttons, impaired gait/balance)

(C) Vocational (eg, inability or reduced ability to perform job duties, challenges obtaining/maintaining employment, avoidance of people)

(D) Psychological (eg, job and social satisfaction, distress related to awareness of TD, loss of sense of purpose, hopelessness, helplessness, frustration, anger, fear, feelings of loss, embarrassment, shame)

(E) Psychiatric disorder (eg, effect of TD on underlying psychiatric condition, compliance with treatment for 
underlying condition, development of other psychiatric symptoms [eg, anxiety] due to TD)

\section{Social Functioning}

Social withdrawal and social isolation are increased in patients with schizophrenia or mood disorders who have antipsychotic-induced TD. ${ }^{29,32}$ These types of negative social effects of TD are experienced by both patients and caregivers. For example, social isolation has been reported by $72.7 \%$ of the patients with TD and $18.2 \%$ of the caregivers, making it among the most commonly reported negative impacts of TD. ${ }^{16}$ In addition, TD in patients with schizophrenia is correlated to decreased likelihood for marriage, ${ }^{32}$ and individuals with orofacial TD symptoms are perceived as being less socially acceptable and as less favorable potential friends and romantic partners. ${ }^{33,34}$

\section{Physical Functioning}

Increased TD severity is associated with greater impact of the disease on multiple aspects of physical functioning, and these effects can be debilitating. ${ }^{16,35,36}$ Difficulty speaking is one of the most commonly reported negative impacts of $\mathrm{TD}$, reported by $72.7 \%$ of the patients and $45.5 \%$ of the caregivers. Respiratory irregularities associated with TD have also been reported in a small proportion of patients hospitalized long term with psychiatric disorders. ${ }^{37,38}$ Other physically limiting consequences of TD can include impaired gait, posture, balance, range of motion, strength, eating/swallowing, damage to teeth due to bruxism, pain associated with abnormal posturing (especially of the neck), and reduced fine motor function (eg, hand dexterity), which can have a profound impact on the performance of activities of daily living, such as grooming, dressing, toileting, bathing, ambulation, and transportation. ${ }^{10,12,38,39}$

\section{Vocational Functioning}

TD is associated with increased likelihood of unemployment and underemployment. For example, in a Malaysian study of 71 patients with antipsychotic-related TD, $75 \%$ of the patients were unemployed, although most patients had only mild psychotic symptoms and had completed secondary education. ${ }^{32}$ Similarly, a prospective cohort study of 739 patients with antipsychotic exposure found that individuals with TD were significantly less likely to be working or studying than those without TD. More than half of patients with TD reported that their employment status was "disabled" $(P=0.0027$ vs patients without TD $){ }^{2}$
Stigma associated with TD can also lead to a perception that individuals with orofacial symptoms are less employable than individuals without $\mathrm{TD}^{33,34}$ Negative effects on employment may also be a result of patients with uncontrolled TD avoiding public places and attempting to hide their abnormal movements. ${ }^{27}$

\section{Psychological Functioning}

TD can cause patients to develop odd postures and movements that can invite ridicule and ostracism. ${ }^{10}$ As a result, many patients with TD report feeling embarrassed by their medical condition (eg, feeling like people are staring at them in public places). ${ }^{10,29,31,39}$ Many patients and caregivers describe emotional and social impacts of TD as the most debilitating features of this disease. ${ }^{29}$ For example, in a series of qualitative interviews, Farber and colleagues ${ }^{16}$ found that unwanted attention related to TD was the most common negative effect of disease, reported by $90.9 \%$ of the patients and $45.5 \%$ of the caregivers In another study, $75.5 \%$ of the patients with TD reported feelings of selfconsciousness or embarrassment related to their involuntary movements. ${ }^{11}$

\section{Underlying Psychiatric Disorders}

Patients with severe or refractory schizophrenia or other psychosis may be prescribed higher doses of antipsychotics, which are known to increase the risk for TD. ${ }^{27,40}$ Use of higher antipsychotic doses may explain observations that TD appears to be associated with a worse long-term disease course of schizophrenia. ${ }^{10,41}$ Thus, studies examining the relationship between severity of TD and severity of schizophrenia can be confounded by antipsychotic dose.

In many cases, doses of antipsychotic medications are reduced in an attempt to prevent or ameliorate symptoms of TD. However, this strategy can result in insufficient control or relapse of the underlying psychiatric illness and may also cause withdrawal dyskinesias. ${ }^{1,19,41,42}$

\section{Time Points for Assessing the Impact of TD}

Recommendation \#4: Assessment of TD impact should be a routine aspect of the evaluation performed at every patient visit because TD impact varies over time and can influence treatment decisions.

The ongoing monitoring of impact is consistent with the American Psychiatric Association recommendation to use the AIMS or a similar instrument to periodically 
monitor for the development of worsening movements in patients treated with antipsychotics. ${ }^{43}$

Frequent assessment of TD impact is important because of the variable and often unstable nature of the condition over time. ${ }^{12}$ Additionally, TD impact may change in response to treatment, external circumstances, or changes in personal situations (eg, employment). Given the potentially broad impact of TD on all aspects of life, the patient's entire health care team should be involved in the assessment process.

Despite the importance of measuring TD impact, there has been limited progress toward implementation of impactspecific instruments or assessment procedures in routine clinical practice. In research settings, several tools have been used to assess the severity of TD symptoms, such as accelerometers, position transducers, electromyography, gait analysis, computerized analysis of motor speech disorders, pegboard and spiral-drawing tests, measurement of hand and postural tremors, isometric strength assessment, cognitionbased assessments, and other TD-specific patient-reported outcome measures. ${ }^{12,44,45}$ However, use of these research tools is limited in clinical settings.

When patients are treated for $\mathrm{TD}$, assessment of changes in the degree of impact in response to treatment should be part of routine clinical evaluations. ${ }^{1}$ In patients with untreated TD, routine monitoring should be performed to quickly identify any worsening of the condition or its impact, which may warrant the initiation of treatment for TD. ${ }^{1,19,26}$

\section{Approaches to Assessing the Impact of TD}

Recommendation \#5: The key domains listed in Recommendation \#3 should be utilized to assess TD impact. When assessing TD impact, clinicians should consult with the patient directly and/or with caregivers, family, and friends, especially when the individual has limited insight into their abnormal movements and their impact on function.

The key domains proposed here are more specific than AIMS items 9 and 10, which are global measures of incapacitation and distress. ${ }^{46}$ Item 9 is a global judgment of incapacitation due to abnormal movements, rated from $0=$ none/ normal to $4=$ severe, and item 10 rates the patient's awareness of abnormal movements and level of distress due to the abnormal movements on an adjusted scale from $0=$ no awareness to $4=$ awareness with severe distress. ${ }^{47}$

\section{Initiating Treatment of TD}

Recommendation \#6: A shared decision to initiate treatment of TD, as well as the urgency with which to initiate treatment, should consider the degree of impact.

Treatment of TD is a shared decision between the patient, his or her caregiver(s), and the clinician to be evaluated on a case-by-case basis, considering the levels of distress and impairment to the patient resulting from dyskinetic movements. ${ }^{11}$ Clinicians should be aware that each person may be impacted in different ways than other individuals experiencing similar degrees of abnormal movements, depending upon each individual's personal situation. ${ }^{24}$ The impact of TD for each individual may change over time (eg, for a person who had not planned to have a job but then received an employment opportunity). ${ }^{24}$

Even if a patient's dyskinetic movements are considered mild, the impact of TD may be significant to that individual. ${ }^{24}$ Other elements to consider when pursuing TD treatment include the individual's risk for worsening TD and the impact of treatment on the underlying psychiatric illness. ${ }^{10,11}$ Treatment of TD is appropriate when the impact of TD outweighs the risk of treatment. ${ }^{7}$ Examples of factors that may worsen TD and TD impact include antipsychotic medication, anticholinergic medication, and external circumstances/stressors (eg, the current global pandemic). ${ }^{47}$

\section{Discussion}

TD can have a profound negative impact on many different functional domains. Physical symptoms of TD can impact communication; swallowing, eating, dentition; gait and posture; and ability to perform activities of daily living (eg, writing, use of technology, self-care). ${ }^{19}$ These physical effects can significantly impact social relationships $^{32-34}$ and the ability to obtain or maintain employment $^{2}$; can lead to feelings of shame, embarrassment, and helplessness; and can impair psychological functioning. ${ }^{11}$ TD can also have a negative impact on a patient's underlying psychiatric illness.

\section{Current Unmet Needs and Future Directions}

There is currently no single clinical tool that can facilitate measuring of the impact of TD. ${ }^{12}$ Current methods used for the evaluation of TD movements and impact are often informal and their use is restricted due to lack of time 
during patient visits and the perception of added burden when using standardized rating scales.

A concise easy-to-use validated instrument would be valuable for the assessment of the potentially broadranging social, physical, vocational, psychological, and psychiatric impacts of TD. Now that approved pharmacotherapies are available to treat $\mathrm{TD}$, a validated tool would also be helpful to assess whether the impact of TD is reduced in treated patients and whether these improvements are maintained over time. Having a baseline assessment using this type of tool would aid in decisions about when to initiate or modify treatment of TD. ${ }^{12}$

To fill this unmet need, a simple TD impact scale could be developed using the key domains identified by this consensus panel (ie, social, physical, vocational, and psychological functioning and the impact of TD on the underlying psychiatric disorder). Ideally, such an instrument would be relatively short (eg, with 1-2 questions per domain) and would have uniform rating scales for each question based on either frequency or severity of impact.

\section{Conclusion}

Overall, the recommendations presented here are intended to provide consensus-based guidance on assessment of the impact of TD across a broad range of functional domains, including social, physical, vocational, and psychological functioning, and the impact of TD on the underlying psychiatric disorder. Timely assessment of TD impact has the potential to better inform shared clinical treatment decision-making to reduce the overall impact of TD, and to hopefully improve long-term patient outcomes.

\section{Abbreviations}

AIMS, Abnormal Involuntary Movement Scale; DISCUS, Dyskinesia Identification System: Condensed User Scale; mCDQ-24, modified Craniocervical Dystonia Questionnaire; TD, tardive dyskinesia; TDRS, Tardive Dyskinesia Rating Scale.

\section{Acknowledgments}

Teva Pharmaceuticals provided financial support to Interactive Forums to conduct the two consensus panel meetings but did not participate in those meetings. Teva Pharmaceuticals also provided financial support to Cello Health Communications/ MedErgy but did not contribute to the consensus process or manuscript preparation. Emily C. Barker, PhD, Stephen D. Lande, PhD, and Jessica McGrory (Interactive Forums, Inc.) assisted with facilitating the consensus panel process and summarizing the key findings. Cherie Koch, $\mathrm{PhD}$ (Cello Health Communications/MedErgy) provided editorial assistance in the preparation of this report.

\section{Author Contributions}

All authors made substantial contributions to conception, design, and interpretation; took part in drafting the article or revising it critically for important intellectual content; agreed to submit to the current journal; gave final approval of the version to be published; and agree to be accountable for all aspects of the work.

\section{Funding}

Preparation of this manuscript was supported by an independent medical grant sponsored by Teva Pharmaceuticals. The sponsor had no involvement in the development of these consensus recommendations or in development of this manuscript.

\section{Disclosure}

Richard Jackson: Conducted research for AbbVie, Avanir, Emalex, Janssen, Neurocrine, Otsuka, Purdue, and Sunovion; served as a consultant/speaker bureau participant for AbbVie, Alkermes, Janssen, Ironshore, Intracellular, Lundbeck, Noven, Otsuka, Purdue, Sunovion, Supernus, and Teva; and assisted in report writing for Cello Health Communications/MedErgy and was involved in honorarium for panel discussion that helped to develop the manuscript for Interactive Forums, Inc. Matthew N. Brams: Served as a speaker bureau participant for Intra-Cellular Therapies, Ironshore, Neos, Otsuka, and Tris Pharmaceuticals. Leslie Citrome: In the past 12 months, served as a consultant for AbbVie, Acadia, Alkermes, Allergan, Angelini, Astellas, Avanir, Axsome, BioXcel, Boehringer Ingelheim, Cadent Therapeutics, Eisai, Impel, Intra-Cellular Therapies, Janssen, Karuna, Lundbeck, Lyndra, Medavante-ProPhase, Merck, Neurocrine, Noven, Otsuka, Ovid, Relmada, Sage, Sunovion, Teva, and the University of Arizona; and provided one-off ad hoc consulting for individuals/entities conducting marketing, commercial, or scientific scoping research; served as a speaker for AbbVie, Acadia, Alkermes, Allergan, Angelini, Eisai, Intra-Cellular Therapies, Janssen, Lundbeck, Neurocrine, Noven, Otsuka, Sunovion, Takeda, Teva, and CME activities organized by medical education companies such as Medscape, NACCME, NEI, Vindico, and universities and professional organizations/societies; owns stock (small number of shares of common stock) in Bristol Myers Squibb, Eli Lilly, Johnson \& Johnson, Merck, and 
Pfizer purchased $>10$ years ago; received royalties from Wiley (Editor-in-Chief, International Journal of Clinical Practice, through the end of 2019), UpToDate (reviewer), Springer Healthcare (book), Elsevier (Topic Editor, Psychiatry, Clinical Therapeutics); and reported personal fees for participating in a virtual consensus conference for Interactive Forums, Inc. and nonfinancial support for editorial/writing support for Cello Health Communications/ MedErgy. Amber Hoberg: served as a speaker and/or on advisory boards for Acadia, Avanir, Intra-Cellular Therapies, and Teva. Stuart H. Isaacson: received honoraria for CME; served as a consultant or promotional speaker for or received research grants from AbbVie, Acadia, Acorda, Adamas, Addex, Affiris, Alexva, Allergan, Amarantus, Amneal, Aptinyx, Axial, Axovant, Benevolent, Biogen, Britannia, Cadent, Cala, Cerecor, Cerevel, Cipla, Eli Lilly, Enterin, GE Healthcare, Global Kinetics, Impax, Impel, Intec Pharma, Ipsen, Jazz, Kyowa, Lundbeck, Merz, Michael J. Fox Foundation, Mitsubishi Tanabe, Neuralys, Neurocrine, Neuroderm, Parkinson Study Group, Pharma2B, Prilenia, Promentis, Revance, Roche, Sanofi, Sunovion, Sun Pharma, Supernus, Teva, Theravance, and $\mathrm{UCB}$; and received personal fees from Interactive Forums, Inc. (Teva) and nonfinancial support from Cello Health Communications/MedErgy. John Kane: In the past 12 months, served as a consultant to or received honoraria for lectures from Acadia, Alkermes, Allergan, Dainippon Sumitomo, Intra-Cellular Therapies, Janssen, Johnson \& Johnson, LB Pharma, Lundbeck, Merck, Minerva, Neurocrine, Otsuka, Reviva, Roche, Saladex, Sunovion, Takeda, and Teva; received grants from Otsuka, Lundbeck, Sunovion, and Janssen; and is a shareholder of LB Pharma, North Shore Therapeutics, and The Vanguard Research Group. Rajeev Kumar: In the past 12 months, received honoraria for CME; served as a consultant or promotional speaker for or received research grants from AbbVie, Acadia, Acorda, Amneal, Biogen, Cala, Cerevel, Eli Lilly, Enterin, Global Kinetics, Impel, Kyowa, Mitsubishi Tanabe, Neuraly, Neurocrine, Neuroderm, Parkinson Study Group, Pharma2B, Prilenia, Revance, Roche, Sage Therapeutics, Supernus, Teva, and US World Meds. The authors report no other conflicts of interest in this work.

\section{References}

1. Caroff SN, Citrome L, Meyer J, et al. A modified Delphi consensus study of the screening, diagnosis, and treatment of tardive dyskinesia. J Clin Psychiatry. 2020;81(2):19cs12983. doi:10.4088/ JCP.19cs12983
2. Caroff SN, Yeomans K, Lenderking WR, et al. RE-KINECT: a prospective study of the presence and healthcare burden of tardive dyskinesia in clinical practice settings. J Clin Psychopharmacol. 2020;40(3):259-268. doi:10.1097/JCP.0000000000001201

3. Cornett EM, Novitch M, Kaye AD, Kata V, Kaye AM. Medicationinduced tardive dyskinesia: a review and update. Ochsner J. 2017;17 (2):162-174.

4. Wilcox PG, Bassett A, Jones B, Fleetham JA. Respiratory dysrhythmias in patients with tardive dyskinesia. Chest. 1994;105 (1):203-207. doi:10.1378/chest.105.1.203

5. Sreeram V, Shagufta S, Kagadkar F. Role of vesicular monoamine transporter 2 inhibitors in tardive dyskinesia management. Cureus. 2019;11(8):e5471. doi:10.7759/cureus.5471

6. Waln O, Jankovic J. An update on tardive dyskinesia: from phenomenology to treatment. Tremor Other Hyperkinet Mov (N Y). 2013;3:1-11.

7. Bhidayasiri R, Boonyawairoj S. Spectrum of tardive syndromes: clinical recognition and management. Postgrad Med J. 2011;87 (1024):132-141. doi:10.1136/pgmj.2010.103234

8. Ali Z, Roque A, El-Mallakh RS. A unifying theory for the pathoetiologic mechanism of tardive dyskinesia. Med Hypotheses. 2020;140:109682. doi:10.1016/j.mehy.2020.109682

9. Woerner MG, Alvir JM, Saltz BL, Lieberman JA, Kane JM. Prospective study of tardive dyskinesia in the elderly: rates and risk factors. Am J Psychiatry. 1998;155(11):1521-1528. doi:10.1176/ ajp.155.11.1521

10. Caroff SN, Ungvari GS, Owens DG. Historical perspectives on tardive dyskinesia. J Neurol Sci. 2018;389:4-9. doi:10.1016/j. jns.2018.02.015

11. Caroff SN. Overcoming barriers to effective management of tardive dyskinesia. Neuropsychiatr Dis Treat. 2019;15:785-794. doi:10.21 47/NDT.S196541

12. Strassnig M, Rosenfeld A, Harvey PD. Tardive dyskinesia: motor system impairments, cognition and everyday functioning. CNS Spectr. 2018;23(6):370-377. doi:10.1017/ S1092852917000542

13. Anderson KE, Stamler D, Davis MD, et al. Deutetrabenazine for treatment of involuntary movements in patients with tardive dyskinesia (AIM-TD): a double-blind, randomised, placebo-controlled, Phase 3 trial. Lancet Psychiatry. 2017;4(8):595-604. doi:10.1016/ S2215-0366(17)30236-5

14. Fernandez HH, Stamler D, Davis MD, et al. Long-term safety and efficacy of deutetrabenazine for the treatment of tardive dyskinesia. J Neurol Neurosurg Psychiatry. 2019;90(12):1317-1323. doi:10.11 36/jnnp-2018-319918

15. Fernandez HH, Factor SA, Hauser RA, et al. Randomized controlled trial of deutetrabenazine for tardive dyskinesia: the ARM-TD study. Neurology. 2017;88(21):2003-2010. doi:10.1212/WNL.0000000000 003960

16. Farber R, Sparta D, Gauthier M, Witherspoon B, O'Brien C, Evans C Tardive dyskinesia: patient and caregiver perspectives on signs, symptoms, and impact. Poster presented at: Psych Congress; September 16-19, 2017; New Orleans, LA.

17. Simpson GM, Lee JH, Zoubok B, Gardos G. A rating scale for tardive dyskinesia. Psychopharmacology (Berl). 1979;64(2): 171-179. doi:10.1007/BF00496058

18. Sprague RL, Kalachnik JE, Slaw KM. Psychometric properties of the Dyskinesia Identification System: Condensed User Scale (DISCUS). Ment Retard. 1989;27(3):141-148.

19. Jain R, Correll CU. Tardive dyskinesia: recognition, patient assessment, and differential diagnosis. J Clin Psychiatry. 2018;79(2): nu17034ah1c. doi:10.4088/JCP.nu17034ah1c

20. Hauser RA, Meyer JM, Factor SA, et al. Differentiating tardive dyskinesia: a video-based review of antipsychotic-induced movement disorders in clinical practice. CNS Spectr. 2020:1-10. doi:10.1017/ S109285292000200X 
21. Caroff SN, Hurford I, Lybrand J, Campbell EC. Movement disorders induced by antipsychotic drugs: implications of the CATIE schizophrenia trial. Neurol Clin. 2011;29(1):127-148. doi:10.1016/j. ncl.2010.10.002

22. Kremens DE. Earlier diagnosis of tardive dyskinesia. $J$ Clin Psychiatry. 2019;81(1):NU18041BR1C. doi:10.4088/JCP.NU18041B R1C

23. Schooler NR, Kane JM. Research diagnoses for tardive dyskinesia. Arch Gen Psychiatry. 1982;39(4):486-487. doi:10.1001/archpsyc.19 82.04290040080014

24. Bakke M, Henriksen T, Biernat HB, Dalager T, Møller E. Interdisciplinary recognizing and managing of drug-induced tardive oromandibular dystonia: two case reports. Clin Case Rep. 2018;6 (11):2150-2155. doi:10.1002/ccr3.1548

25. Vasan S, Padhy RK. Tardive dyskinesia. In: StatPearls [Internet]. FL: StatPearls Publishing; 2021. Available from: https://www.ncbi. nlm.nih.gov/books/NBK448207/.

26. Correll CU, Kane JM, Citrome LL. Epidemiology, prevention, and assessment of tardive dyskinesia and advances in treatment. J Clin Psychiatry. 2017;78(8):1136-1147. doi:10.4088/JCP.tv17016ah4c

27. Citrome L, Saklad SR. Revisiting tardive dyskinesia: focusing on the basics of identification and treatment. J Clin Psychiatry. 2020;81(2): TV18059AH3C. doi:10.4088/JCP.TV18059AH3C

28. American Psychiatric Association. Tardive dyskinesia: summary of a task force report of the American Psychiatric Association. By the Task Force on Late Neurological Effects of Antipsychotic Drugs. Am J Psychiatry. 1980;137(10):1163-1172. doi:10.1176/ajp.137.10.1163

29. McEvoy J, Gandhi SK, Rizio AA, et al. Effect of tardive dyskinesia on quality of life in patients with bipolar disorder, major depressive disorder, and schizophrenia. Qual Life Res. 2019;28(12):3303-3312. doi:10.1007/s11136-019-02269-8

30. Wu JQ, Chen DC, Xiu MH, et al. Tardive dyskinesia is associated with greater cognitive impairment in schizophrenia. Prog Neuropsychopharmacol Biol Psychiatry. 2013;46:71-77. doi:10.10 16/j.pnpbp.2013.06.013

31. Hauser RA, Truong D. Tardive dyskinesia: out of the shadows. J Neurol Sci. 2018;389:1-3. doi:10.1016/j.jns.2018.02.009

32. Othman Z, Ghazali M, Razak AA, Husain M. Severity of tardive dyskinesia and negative symptoms are associated with poor quality of life in schizophrenia patients. Int Med J. 2013;20(6):677-680.

33. Ayyagari R, Goldschmidt D, Mu F, Caroff SN, Carroll B. An experimental study to assess the professional and social consequences of tardive dyskinesia. CNS Spectr. 2020;25(2):275-276.

34. Boumans CE, de Mooij KJ, Koch PA, Van't Hof MA, Zitman FG. Is the social acceptability of psychiatric patients decreased by orofacial dyskinesia? Schizophr Bull. 1994;20(2):339-344. doi:10.1093/ schbul/20.2.339

35. Cutler AJ, Caroff SN, Shalhoub H, Lenderking WR, Franey E, Yonan C. Health-related quality of life in patients with possible tardive dyskinesia based on patient and clinician assessments. CNS Spectr. 2020;25(2):268-269. doi:10.1017/S1092852920000231
36. Rittmannsberger $H$. Ten year outcome of tardive dyskinesia during continuous treatment with first generation antipsychotics. Psychiatr Danub. 2008;20(4):461-465.

37. Yassa R, Lal S. Respiratory irregularity and tardive dyskinesia. A prevalence study. Acta Psychiatr Scand. 1986;73(5):506-510. doi:10.1111/j.1600-0447.1986.tb02717.x

38. Yassa R, Jones BD. Complications of tardive dyskinesia: a review. Psychosomatics. 1985;26(4):305-307, 310, 312-313. doi:10.1016/ S0033-3182(85)72863-0

39. Yassa R. Functional impairment in tardive dyskinesia: medical and psychosocial dimensions. Acta Psychiatr Scand. 1989;80(1):64-67. doi:10.1111/j.1600-0447.1989.tb01301.x

40. Jankelowitz SK. Treatment of neurolept-induced tardive dyskinesia. Neuropsychiatr Dis Treat. 2013;9:1371-1380. doi:10.2147/NDT. S30767

41. Ascher-Svanum H, Zhu B, Faries D, Peng X, Kinon BJ, Tohen M. Tardive dyskinesia and the 3-year course of schizophrenia: results from a large, prospective, naturalistic study. J Clin Psychiatry. 2008;69(10):1580-1588. doi:10.4088/JCP.v69n1008

42. Adrianzé C, Arango-Dávila C, Araujo DM, et al. Relative association of treatment-emergent adverse events with quality of life of patients with schizophrenia: post hoc analysis from a 3-year observational study. Hum Psychopharmacol. 2010;25(6):439-447. doi:10.1002/ hup. 1143

43. American Psychiatric Association. Treatment of patients with schizophrenia: tardive dyskinesia. Available from: https://www.guideline central.com/shop/treatment-patients-schizophrenia-tardive-dyskinesia -guidelines. Accessed February 22, 2021.

44. Müller J, Wissel J, Kemmler G, et al. Craniocervical Dystonia Questionnaire (CDQ-24): development and validation of a disease-specific quality of life instrument. J Neurol Neurosurg Psychiatry. 2004;75(5):749-753. doi:10.1136/jnnp.2003.013441

45. Dean CE, Russell JM, Kuskowski MA, Caligiuri MP, Nugent SM. Clinical rating scales and instruments: how do they compare in assessing abnormal, involuntary movements? J Clin Psychopharmacol. 2004;24(3):298-304. doi:10.1097/01.jcp.000012 5681.97466.e7

46. Kane JM, Correll CU, Nierenberg AA, Caroff SN, Sajatovic M, for Tardive Dyskinesia Assessment Working Group. Revisiting the abnormal involuntary movement scale: proceedings from the Tardive Dyskinesia Assessment Workshop. J Clin Psychiatry. 2018;79(3):17cs11959. doi:10.4088/JCP.17cs11959

47. Citrome L. Treating TD in the COVID-19 era: 5 steps to success. Psychiatry \& Behavioral Health Learning Network; 2020. Available from: https://www.psychcongress.com/multimedia/treating-td-covid -19-era-5-steps-success. Accessed November 2, 2020.
Neuropsychiatric Disease and Treatment

\section{Publish your work in this journal}

Neuropsychiatric Disease and Treatment is an international, peerreviewed journal of clinical therapeutics and pharmacology focusing on concise rapid reporting of clinical or pre-clinical studies on a range of neuropsychiatric and neurological disorders. This journal is indexed on PubMed Central, the 'PsycINFO' database and CAS, and is the official journal of The International Neuropsychiatric Association (INA). The manuscript management system is completely online and includes a very quick and fair peer-review system, which is all easy to use. Visit http://www.dovepress.com/testimonials.php to read real quotes from published authors. 